\title{
A case report of a high division of Extensor digitorum longus
}

\author{
Eshwara $\mathrm{JM}^{1}$ *, Kosgallana $\mathrm{EW}^{1}$, Dissanayake $\mathrm{JK}^{1}$ \\ ${ }^{I}$ Department of Anatomy, Faculty of Medicine, University of Peradeniya. \\ *janith9eshwara@gmail.com
}

Extensor digitorum longus (EDL) is a muscle of the extensor compartment of the leg. It arises from inferior surface of the lateral condyle of tibia, upper three quarter of extensor surface of fibula, anterior surface of interosseus membrane and deep surface of deep fascia, becomes tendinous in the leg, then passes deep to both superior and inferior extensor retinacula. In the dorsum of the foot EDL tendon usually divides into 4 slips which passes forward to $2 \mathrm{nd}$, $3 \mathrm{rd}$, 4 th, 5 th toes to form extensor expansions. It is an extensor of the toes as well as the ankle joint. While it is essential for the gait, it has an importance in tendon reconstruction surgeries. During routine dissections a variation of EDL muscle was noted. It originated from lateral tibial condyle, deep fascia, fibula, and interosseus membrane. The muscle belly divided into 2 as it became tendinous in the proximal leg. The 2 tendons passed under both superior and inferior extensor retinacula. The medial tendon gave slips to $2^{\text {nd }}$ and $3^{\text {rd }}$ toes while the lateral tendon gave slips to 4th and 5th toes. The lateral tendon additionally had an inter-tendinous connection with the tendon of Peroneus tertius. Variations of EDL are not commonly reported. This high bifurcation of EDL may have implications in surgery, especially during reconstruction following trauma. 